DOI: $10.3901 / J M E .2020 .18 .226$

\title{
大流量压电式高速开关阀设计与仿真测试
}

\author{
俞军涛 $^{1}$ 焦宗夏 $^{2}$ 吴 帅 ${ }^{2}$ \\ (1. 山东大学(威海)机电与信息工程学院 威海 264200; \\ 2. 北京航空航天大学自动化科学与电气工程学院 北京 100191)
}

\begin{abstract}
摘要: 针对目前高速开关阀快速性的不足及大流量的需求, 利用压电晶体响应快速的特点, 设计一种压电直动式高速开关 阀, 采用压电执行器直接驱动锥阀式阀芯结构, 控制开关阀开启和关闭。设计桥式位移放大结构, 放大压电执行器的输出 位移, 提高开关阀的控制流量。为补偿锥阀式阀芯结构开启、关闭过程中的液动力波动, 提出锥阀+滑阀的组合式阀芯结 构, 建立其液动力模型, 分析开关阀启闭过程中的负载力变化。建立压电直动式高速开关阀的数学模型, 结合 AMESim 仿真软件分析、优化影响其性能的设计参数。搭建压电高速开关阀静态流量及动态启闭特性的试验测试平台, 测试开关阀 流量特性、启闭时间等性能指标，分析 PWM 控制载波频率及占空比与开关阀静态、动态流量之间的关系。基于设计参数 仿真和实物样机试验测试结果表明, 设计的压电式开关阀在压力 $10 \mathrm{MPa}$ 下, 输出流量 $17.4 \mathrm{~L} / \mathrm{min}$, 开启时间 $1.05 \mathrm{ms,}$ 关闭时间 $0.85 \mathrm{~ms}$, 实现大流量与快速性的要求。研究工作可为高速开关阀精确流量控制提供理论基础, 对于拓展数字液 压技术的应用具有重要意义。
\end{abstract}

关键词: 压电执行器; 位移放大; 高速开关阀; 流量特性; 启闭时间

中图分类号: TH137

\section{Design, Simulation and Test of High-flow High-speed on/off Valve Driven by Piezoelectric}

\author{
YU Juntao ${ }^{1}$ JIAO Zongxia ${ }^{2}$ WU Shuai ${ }^{2}$ \\ (1. School of Mechanical and Information Engineering, Shandong University(Weihai), Weihai 264200; \\ 2. School of Automation Science and Electrical Engineering, Beihang University, Beijing 100191)
}

\begin{abstract}
A piezoelectric (PZT) direct-acting high-speed switching valve is designed in order to improve the lack of rapidity and large flow. In view of the fast response of PZT crystal, the PZT actuator is used to drive the conical valve core structu re directly, which open and close the on/off valve. A bridge-type displacement amplification structure is designed to amplify the displacement output of PZT actuator, which increases the control flow of the switching valve. A combined structure of cone valve and slide valve is proposed to compensate the hydrodynamic fluctuation during the opening and closing process of PZT switching valve. The hydrodynamic model is established to analyze the change of fluid load force. The mathematical model of PZT high-speed on/off valve is established, and the design parameters affecting its performance are analyzed and optimized by AMESim simulation software. The test platform for static flow rate and dynamic open-close characteristics of PZT high-speed on/off valve is built. The PWM driving and control methods are studied based on the performance indexes such as flow characteristics, opening and closing time. The relationship between carrier frequency, duty cycle and static, dynamic flow of on-off valve is analyzed. The simulation and experimental results based on the prototype and design parameters show that the designed PZT on/off valve has a flow rate of $17.4 \mathrm{~L} / \mathrm{min}$, an opening time of $1.05 \mathrm{~ms}$ and a closing time of $0.85 \mathrm{~ms}$ at the pressure of $10 \mathrm{MPa}$. The requirement of high speed and large flow is realized. The research work provides a theoretical basis for the accurate flow control of high speed on/off valve and is of great significance for the application of digital hydraulic technology.
\end{abstract}

Key words: PZT actuator; displacement amplification; high-speed on/off valve; flow characteristics; opening and closing time

* 国家自然科学基金(51235002)和山东省重点研发计划(2019JZZY010337)

资助项目。20190918 收到初稿, 20200331 收到修改稿 


\section{0 前言}

数字控制技术作为一种新型的电液控制技术, 相比传统的比例、伺服控制技术具有可靠性高、精 度高、效率高以及控制简单、灵活等优点。开关阀 作为数字液压控制系统中关键的核心控制元件, 具 有体积小、响应快、控制灵活和抗污染能力强的优点, 非常适合煤炭、锻压和工程机械等恶劣环境下工作; 其中高速开关阀，通过提高 PWM 控制载波的频率， 亦可实现高速、高精度的液压伺服控制 ${ }^{[1-2]}$ 。

传统的开关阀大多采用电磁铁作为电-机械转 换器, 为提高开关阀的快速性, 目前的研究方向大 多集中在强力高速电磁铁。然而电磁铁受体积、电 磁力及运动部件惯性等因素的影响, 其响应速度受 到制约, 且存在体积大、易发热、抗电磁干扰能力 差的问题 ${ }^{[3]}$ 。采用音圈电机、磁致伸缩及压电材料 驱动的高速开关阀成为目前研究的方向 ${ }^{[4-8]}$, 其中压 电材料是一种电致伸缩材料, 利用压电晶体材料的 逆压电效应设计的压电执行器具有体积小、响应迅 速的优点, 采用压电执行器作为电-机械转换器可以 大大提高开关阀的响应能力; 然而压电执行器输出 位移极小, 其应变只有 $0.1 \%$, 即使采用压电叠堆结 构, 其最大输出位移也只有几十微米, 远达不到驱 动阀芯位移的要求 ${ }^{[9-10]}$ 。

针对压电材料响应快速、输出力大及位移极小 的特点, 各种位移放大结构以及采用新原理, 新结 构的开关阀得到广泛的研究 ${ }^{[1-15]}$ 。吉林大学的沈传 亮等 ${ }^{[16]}$ 提出一种杜杆式位移放大结构, 实现 10 的 位移放大倍数, 但铰链连接处的摩擦和间隙大大降 低了系统整体的精度, 且放大位移输出端轨迹为弧 线, 系统同轴度差; 北京航空航天大学俞军涛等 ${ }^{[17]}$ 提出一种基于柔性铰链的膜片式活塞结构, 相比传 统活塞, 可实现运动过程中的零摩擦和零泄漏, 并 将其用于液压微位移放大结构中，但系统的装配及 维护复杂、困难; 浙江大学欧阳小平等 ${ }^{[18]}$ 利用压电 执行器输出力大的特点, 提出一种基于冲量定理的 撞击式开关阀结构, 实现开关阀的快速开启和关闭, 但冲击带来系统可靠性差，使用寿命低的问题。

柔性铰链结构具有结构简单、运动平稳、无 摩擦和间隙以及分辨率、精度高的优点, 基于柔 性铰链的位移放大结构, 能有效放大压电执行器 的输出位移。本文针对压电叠堆式驱动器的特点, 设计菱形位移放大结构放大其输出位移, 驱动雉 阀式阀芯运动, 控制开关阀开启及关闭; 在此基
础上研究压电式高速开关阀的设计、建模、控制 和测试方法, 并对其静、动态性能进行仿真分析 及试验测试。

\section{1 结构及工作原理}

\section{1 结构}

所设计的压电直动式高速开关阀整体结构如图 1 所示, 采用菱形位移放大结构放大压电执行器的 输出位移, 直接驱动雉阀式阀芯结构, 控制阀口的 开启和关闭。

\section{2 工作原理}

在图 1 所示开关阀结构原理示意图中，阀芯采 用常闭型雉阀结构; 位移放大结构与压电叠堆采用 过盈配合，使之具有一定的预压力，通过调节右端 的调零螺栓实现阀口及流量初始零位的调节; 当脉 冲为高电平时, 压电执行器伸长, 驱动位移放大结 构带动阀芯向右运动, 克服零位预紧力、变形应力 和液动力等, 阀口逐渐打开, $\mathrm{P}$ 与 $\mathrm{A}$ 相连，直到阀 口完全打开。当脉冲控制信号为低电平时，压电执 行器输出位移减小(收缩), 阀芯在位移放大结构变 形应力、预紧力及液压力的作用下，向左运动，堵 住 P、A 之间连接，阀口关闭，在预紧力的作用下， 紧紧密封，基本实现零泄漏。

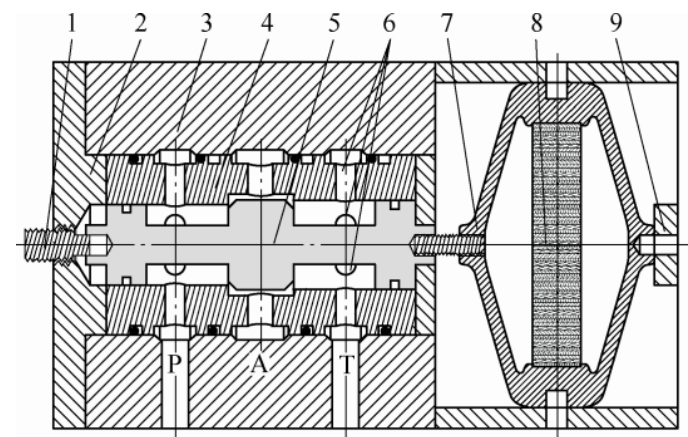

图 1 压电直驱式高速开关阀结构原理示意图

1. 电浴流传感器探头; 2 . 左端盖; 3. 阀体; 4. 阀套; 5. 阀芯; 6. 阀套径向孔； 7. 放大结构; 8. PZT; 9. 固定、调零装置

\section{2 建模及设计}

\section{1 压电执行器模型及仿真}

单片压电陶瓷厚度通常只有 $0.5 \mathrm{~mm}$, 其应变量 为 $1 \%$, 输出位移极小 $(0.5 \mu \mathrm{m})$; 采用机械串联、电 气并联的叠堆式结构可以大大增加压电执行器的输 出位移, 但会降低执行器的刚度。选用 PI 公司的压 电执行器, 基本参数如表 1 所示。 
表 1 压电执行器参数

\begin{tabular}{lc}
\hline \multicolumn{1}{c}{ 参数 } & 数值 \\
\hline 外形尺寸 $/ \mathrm{mm} \times \mathrm{mm} \times \mathrm{mm}$ & $32 \times 10 \times 8$ \\
截面积 $\mathrm{A} / \mathrm{mm} \times \mathrm{mm}$ & $8 \times 8$ \\
单片厚度 $\delta / \mathrm{mm}$ & 0.5 \\
最大出力 $F_{m} / \mathrm{kN}$ & 2.4 \\
最大位移 $x_{m} / \mu \mathrm{m}$ & 30 \\
抗压刚度 $K_{p} /(\mathrm{N} / \mu \mathrm{m})$ & 80 \\
抗拉刚度 $K_{t} /(\mathrm{N} / \mu \mathrm{m})$ & 3.5 \\
共振频率 $f / \mathrm{kHz}$ & 40 \\
\hline
\end{tabular}

空载时, 压电晶体输出位移 $\Delta L$ 与驱动控制电压 $V$ 基本成比例, 即

$$
\Delta L=n d_{33} V
$$

式中, $d_{33}$ 为压电材料的压电系数。

压电晶体带载工作时, 由于其自身刚度限制, 需要预加载力, 设计中利用柔性铰链位移放大结构 零位装配时的变形应力作为预紧力, 在此工况下, 其输出位移及输出力受柔性铰链结构的刚度及预载 力大小影响, 加载情况及特性如图 2 所示。

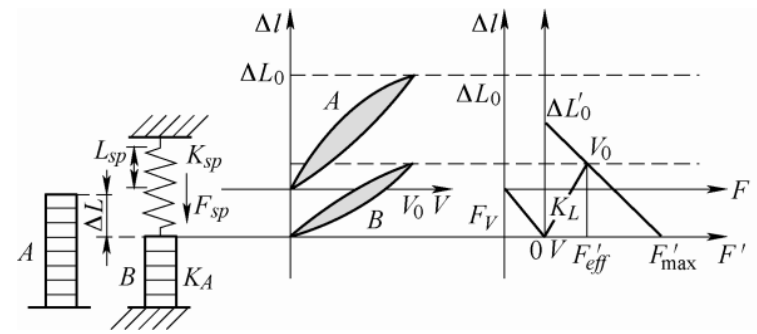

图 2 压电叠堆负载特性

如图 2 所示, 预加载压力为 $F_{s p}$, 则

$$
F_{s p}=K_{s p} \times L_{s p}
$$

式中 $K_{s p}$ 一柔铰放大结构等效刚度;

$L_{s p}$ 一其预压缩量。

此时, 压电晶体被压缩量为 $\Delta L$, 则

$$
\Delta L=F_{s p} / K_{A}
$$

式中, $K_{A}$ 一压电叠堆抗压刚度。

由式(1) (3) 可得: 在柔性铰链位移放大结构的 预载压力下, 驱动电压 $V_{0}$ 一定时, 压电执行器的最 大伸长量 $x_{m}$ (自由端)

$$
x_{m}=n d_{33} V_{0} \times K_{A} /\left(K_{s p}+K_{A}\right)
$$

结合式(1) (4)可得: 压电执行器带载时, 输出位移 $x$ 与驱动力 $F_{p}$ 关系

$$
F_{p}=x_{m} \times\left(K_{s p}+K_{A}\right)-K_{A} \times x
$$

式中 $x$ 一执行器输出位移;

$F_{p}$ —输出驱动力。

\section{2 位移放大结构及仿真分析}

\section{2 .1 位移放大原理模型及分析}

位移放大结构(取其 $1 / 4$ 部分)的原理如图 3 所 示。三角形结构 $A B C$, 初始状态 $A B=l_{1}, B C=d_{1}$, $A B$ 与 $A C$ 夹角为 $\gamma$, 在外力作用下, 三角形变形为 $D B E$, 变形后 $D B=l_{2}, B E=d_{2}, D B$ 与 $D E$ 夹角为 $\beta$; 则横向变形量 $A D=\Delta l=l_{1}-l_{2}$, 纵向变形量 $C E=\Delta d=d_{1}-d_{2}$ ；在应变极小的情况下, $\gamma \approx \beta$, 可得：位移放大倍数 $k=\Delta l / \Delta d \approx \tan \gamma$ 。

\subsection{2 位移放大结构及仿真}

传统铰链连接处存在摩擦及间隙, 小位移时精 度差, 可靠性不高; 柔性铰链具有无间隙、无摩擦、 精度高的优点, 基于位移放大原理, 设计基于柔性 铰链的菱形位移放大结构如图 4 所示。

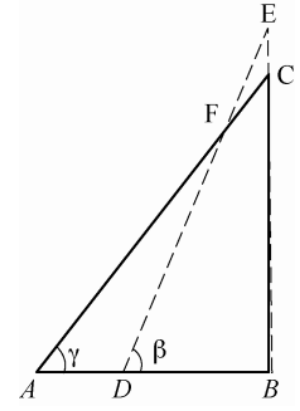

图 3 位移放大原理图

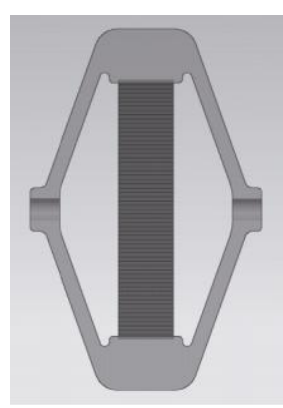

图 4 位移放大结构图
图 4 中，外围菱形框架为位移放大结构，中间 为压电叠堆执行器, 建立位移放大结构的有限元模 型, 并对其进行仿真分析, 结果如图 5 所示。

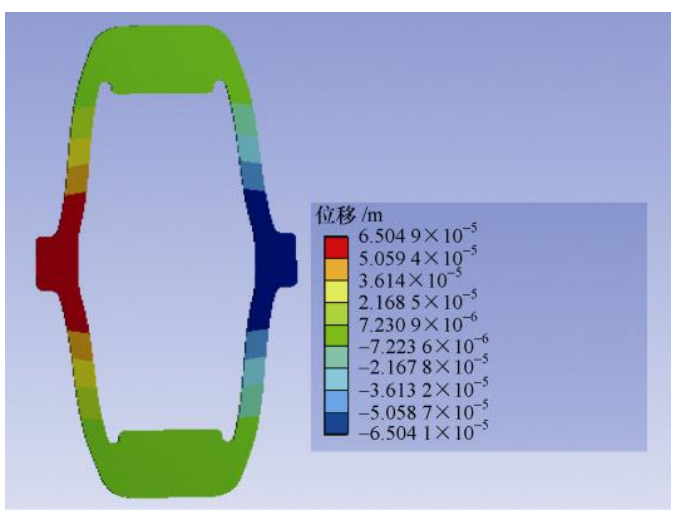

图 5 有限元仿真结果图

菱形位移放大结构外形尺寸为 $48 \mathrm{~mm} \times 21 \mathrm{~mm} \times$ $7 \mathrm{~mm}$; 等效三角结构中, 长边为 $24 \mathrm{~mm}$, 短边为 $5.5 \mathrm{~mm}$ 。选用弹性非常好的铍青铜材料, 在纵向力 $158 \mathrm{~N}$, 横向空载时, 纵向输出位移 $30 \mu \mathrm{m}$, 横向 位移 $130 \mu \mathrm{m}$, 位移放大倍数 4.33(略小于理论计算 值 $24 / 5.5 \approx 4.36$ ), 与理论分析结果基本一致, 同时 
根据模态分析仿真结果, 可得以下结论。

(1) 一阶模态频率为 $1012 \mathrm{~Hz}$, 不低于压电执 行器, 可以保证运动全过程中, 压电叠堆与位移放 大结构的紧密接触。

(2) 等效刚度 $K_{s}$, 横向位移放大的同时, 其输 出力必然减小, 求得其横向等效刚度 $K_{s}=$ $(158 / 4.36) /\left(0.13 \times 10^{-3}\right) \approx 280 \mathrm{kN} / \mathrm{m}$ 。则阀芯最大 开口 $0.13 \mathrm{~mm}$ 时, 回复力为最大开口变形应力 $(280 \times 0.13 \approx 36 \mathrm{~N})$ 和零位预紧力的合力, 足够提供 阀口关闭时的回复力。

\section{3 锥阀结构及分析}

\subsection{1 阀芯结构}

压电执行器出力大、输出位移小, 这要求阀芯 结构具有较大的通流面积, 而由于其控制流量相对 较小, 所以要求泄漏非常小(零泄漏)。

滑阀式结构阀芯凸肩和阀套间存在的配合间 隙, 容易产生泄漏, 而由于压电式开关阀自身的流 量本身就不大, 因此会严重影响阀的控制精度。球 阀式结构由于阀芯及推杆之间连接部分的存在, 导 致阀芯受力不平衡, 增加了电-机械转换器的负载, 同时影响其流量及动态性能; 雉阀式结构的阀芯和 阀套(阀座)之间采用线密封或面密封的形式, 有效 地解决了泄漏问题, 比较适合作为压电式高速开关 阀的阀芯结构, 但其启闭过程中液动力存在波动, 补偿相对困难。

采用雉阀+滑阀的结构形式, 平衡阀芯启闭过程 中的液压力; 采用阀套径向开孔法, 减小稳态径向 液动力。所设计的组合式阀芯结构如图 6 所示, 当 流道内充满流体时, 阀芯左右端所受静态液压力大 小基本相等, 方向相反, 如图 7 所示, 从而大大减 小阀芯运动过程中所受的液压力。

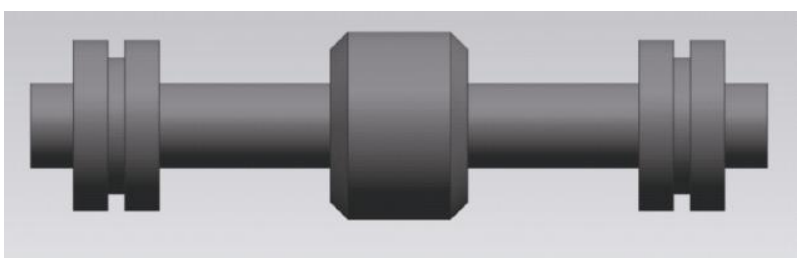

图 6 锥阀阀芯、阀套结构图

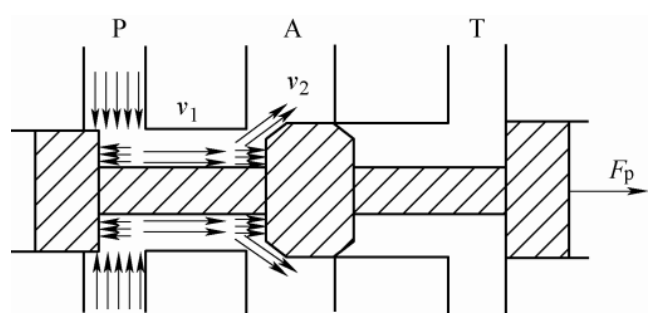

图 7 阀芯开启受力分析图

\subsection{2 液动力分析}

以阀芯作为研究对象, 可得阀芯受力分析图如 图 7 所示, 假设阀芯质量为 $m$, 油液黏性阻尼系数 为 $c$, 复位弹簧(即位移放大结构)等效刚度为 $K_{s}$, 阀芯位移量为 $x$, 雉阀角度为 $\alpha$, 阀芯直径为 $d$, 阀 杆直径为 $d_{0}$, 压力为 $p$, 位移放大式压电执行器输 出力为 $F_{p}$, 稳态液动力为 $F_{d}$, 瞬态液动力为 $F_{z}$ 。

压电执行器在高电平作用下, 阀芯开启, $\mathrm{P}$ 与 $\mathrm{A}$ 相连, $\mathrm{A}$ 与 $\mathrm{T}$ 关闭, 其动力学方程

$$
m \ddot{x}+c \dot{x}+K_{s} x=F_{p}+F_{d}+F_{z}
$$

滑阀所受液压力

$$
F_{d c}=p \pi\left(d^{2}-d_{0}^{2}\right) / 4
$$

外流式雉阀结构稳态液动力

$$
F_{d p}=\rho q_{\mathrm{a}}\left(v_{2} \cos \alpha-v_{1}\right)-p \pi\left(d^{2}-d_{0}^{2}\right) / 4
$$

式中 $v_{1}$ 一进油口流速;

$v_{2}$ 一节流口流速。

$$
v_{2}=C_{v} \sqrt{2(\Delta P) / \rho}
$$

式中 $C_{v}$ 一速度系数;

$v_{1} \ll v_{2}$;

$\Delta P=P_{p}-P_{A}$ 。

雉阀进油口流量连续方程

$$
q_{a}=C_{d} A \sqrt{2 \Delta P / \rho}
$$

式中 $C_{d}$ 一流量系数;

$$
\begin{aligned}
& A \text { 一一通油面积, 雉阀结构中 } \\
& A=\pi d x(1-(x \sin 2 \alpha) / 2 d) \times \sin \alpha
\end{aligned}
$$

由于 $x \ll d$

$$
1-(x \sin 2 \alpha) / 2 d \approx 1
$$

阀芯开启时所受稳态液压力为锥阀和滑阀部分 液压力的合力, 即

$$
F_{d}=F_{d p}+F_{d c}
$$

综合式(7) (11)

$$
F_{d}=C_{v} C_{d} \pi d x \Delta p\left(1-\frac{x \sin \alpha}{2 d}\right) \sin 2 \alpha
$$

瞬态液动力

$$
F_{z}=\rho L C_{d} \pi d \sin 2 \alpha\left(1-\frac{x \sin 2 \alpha}{2 d}\right) \sqrt{2 \Delta p / \rho}
$$

压电执行器低电平时, 在复位弹簧作用下, 阀 芯关闭, $\mathrm{A}$ 与 $\mathrm{T}$ 相连, $\mathrm{P}$ 与 $\mathrm{A}$ 关闭, 动力学方程

$$
m \ddot{x}+c \dot{x}=K_{s} x+F_{d}+F_{z}
$$

出油口流量连续方程

$$
q_{\mathrm{T}}=C_{\mathrm{d}} A \sqrt{2\left(P_{\mathrm{A}}-P_{\mathrm{T}}\right) / \rho}
$$

由式(14)、(15)可以看出, 稳态液动力大小与压 
差 $\Delta p$ 、阀芯直径 $d$ 、阀口开度 $x$ 及阀芯雉角 $\alpha$ 的大 小有关; 暂态液动力方向与阀芯运动方向有关, 开 启时为负, 关闭时为正, 其大小与流量变化及等效 阻尼 $L$ 有关。

\section{4 复位弹簧}

由表 1 所示压电执行器的抗拉、抗压刚度, 可以看 出压电叠堆执行器的结构特点决定其输出推力很大, 但 拉力很小, 无法提供开关阀关闭所需要的驱动力, 因此 通常需要复位弹簧, 弹簧刚度过大, 会降低开关阀的开 启特性，弹簧刚度过小，不利于其关闭特性。

利用位移放大结构自身的刚度特性(仿真等效 刚度 $K_{s}=280 \mathrm{kN} / \mathrm{m}$ ), 针对关闭过程中的液动力变 化, 设置相应的初始预压缩量, 补偿液动力的影响, 可提供阀芯关闭过程的主动回复力, 保证压电执行 器在低脉冲下, 阀口能够快速关闭。

\section{3 仿真与分析}

\section{1 开关阀仿真模型}

在 AMESim 中建立压电式开关阀的模型如图 8 所示。

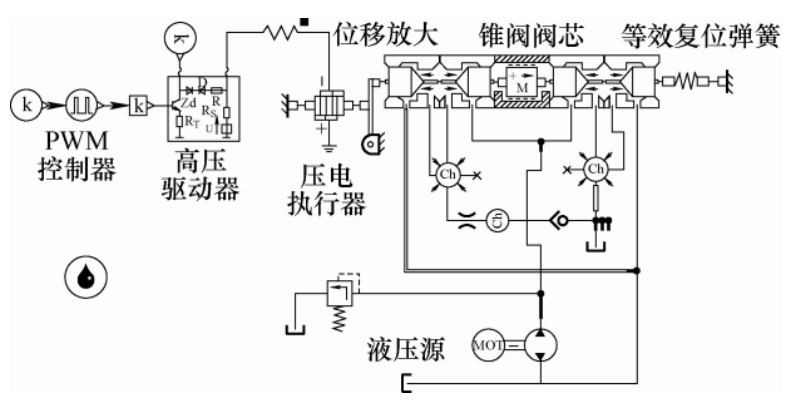

图 8 开关阀仿真模型

图 8 中, 左端输入为一定载波频率下, 占空比 可调的 PWM 脉冲信号, 控制高压压电驱动器; 位 移放大部分采用杜杆式位移放大环节进行代替, 其 放大倍数与菱形位移放大结构相同, 其等效刚度与 最右端的等效复位弹簧刚度相同; 液压部分采用雉 阀结构模型。仿真用到的具体参数详见表 2 。

\section{表 2 开关阀仿真参数}

\begin{tabular}{lc}
\hline \multicolumn{1}{c}{ 参数 } & 数值 \\
\hline 压力 $P / \mathrm{MPa}$ & 10 \\
阀芯直径 $d_{p} / \mathrm{mm}$ & 12 \\
阀芯杆直径 $d_{r} / \mathrm{mm}$ & 5 \\
雉阀角度 $\alpha /\left(^{\circ}\right)$ & 50 \\
阀芯开口 $x_{p} / \mathrm{mm}$ & 0.12 \\
阀芯质量 $m_{p} / \mathrm{g}$ & 14.6 \\
放大结构刚度 $K_{s} /(\mathrm{kN} / \mathrm{m})$ & 280 \\
位移放大倍数 $K$ & 4.3 \\
\hline
\end{tabular}

\section{2 设计参数影响及仿真}

根据上述仿真模型及参数, 仿真计算得到不同 锥阀角度 $\left(25^{\circ} \sim 70^{\circ}\right.$, 每 $5^{\circ}$ 为间隔), 开关阀最大流 量及液压力曲线如图 9 所示。

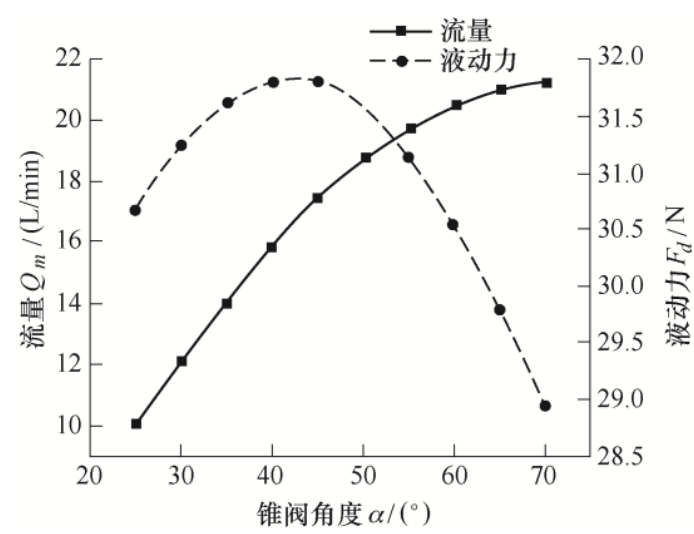

图 9 不同雉阀角度下流量与液压力曲线

仿真计算得到不同阀芯直径 $(8 \sim 14 \mathrm{~mm}$, 每 $1 \mathrm{~mm}$ 为间隔), 开关阀最大流量及液压力曲线如图 10 所示。

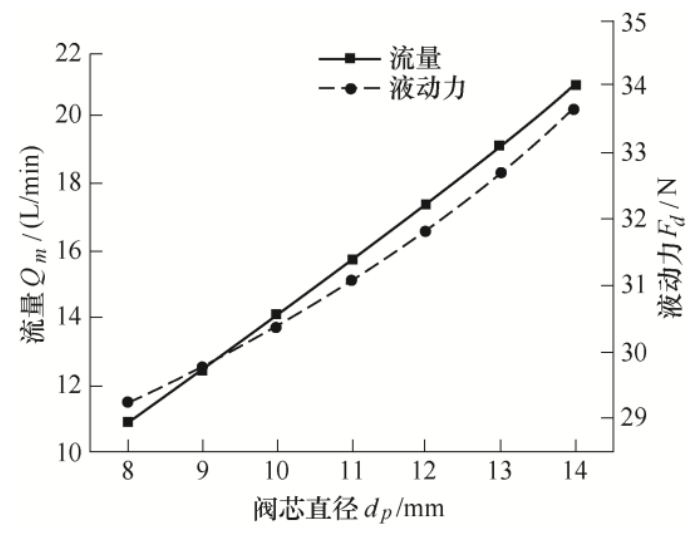

图 10 不同阀芯直径下流量与液压力曲线

由图 9、10 不同阀芯雉角和直径下，开关阀流 量及液压力曲线可以看出: 随着阀芯锥角的增大, 阀口最大通流量增加, 阀芯所受总液力先增大后减 小, 雉角 $\alpha \approx 45^{\circ}$ 时, 液压力最大; 而阀芯直径与开 关阀流量及液动力基本成线性关系; 与式(10)、(14)、 (15)理论分析结果一致。

\section{3 开关阀性能仿真}

取 PWM 信号载波频率 $f=100 \mathrm{~Hz}$, 占空比 $\rho=0.5$, 得一周期内开关阀流量开启、关闭曲线如 图 11 所示。

图 11 中, 纵轴代表开关阀的流量, 横轴代表时 间。由流量启闭曲线可以看出: 所设计的开关阀瞬 态最大流量 $17.4 \mathrm{~L} / \mathrm{min}$, 响应快速; 但由于压电执 行器迟滞特性及阀芯惯性的存在，开关阀死区时间 $0.22 \mathrm{~ms}$; 完全开启时间为 $1.05 \mathrm{~ms}$; 阀口基本完全 关闭所需时间 $0.65 \mathrm{~ms}$ 。 


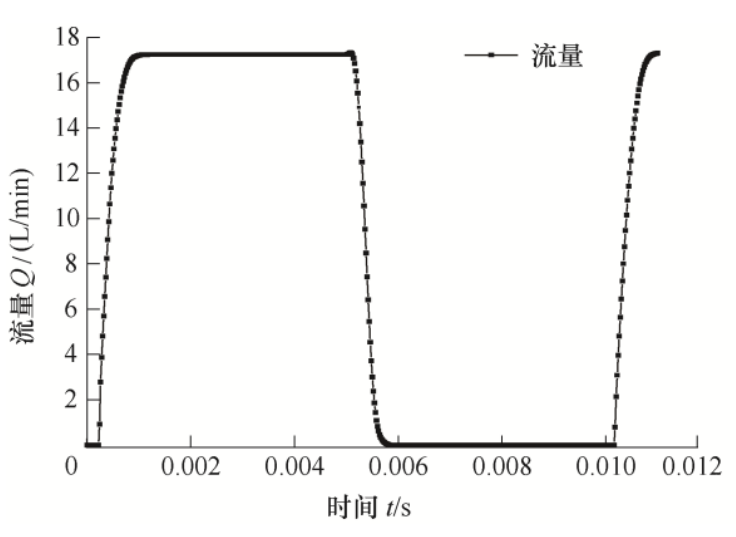

图 11 开关阀流量启、闭仿真曲线

\section{4 试验与测试}

参考表 2 所示的结构参数, 加工、装配好的压 电式高速开关阀样机如图 12 所示。

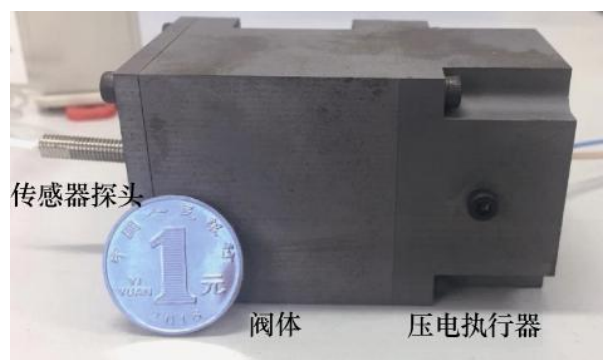

图 12 开关阀样机装配图

开关阀的调制、控制通常采用脉宽式(PWM)方 式, 其基本原理是通过对信号采样及转化, 将模拟 式控制信号转化为频率一定, 占空比不同的调制信 号, PWM 信号为高电平时, 阀口开启; PWM 信号 为低电平时, 阀口关闭。

\section{1 试验平台}

开关阀的试验测试系统如图 13 所示。

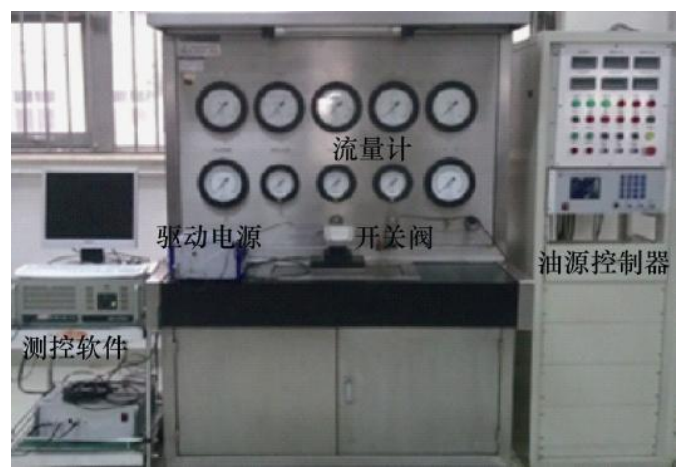

图 13 开关阀试验测试系统

高速开关阀在高频 PWM 信号作用下，阀口液 流通断频率很高, 油液波动不大, 针对阀口不完全 打开的情况, 可以利用一个周期内流经阀口的总流
量 $Q$ 除以周期 $T$, 求得平均控制流量 $q$; 而动态启、 闭特性的测试, 由于高速开关阀开关频率非常高, 动态流量难以精确测试, 由式(10)在压力、结构等 参数一定的情况下，阀口的控制流量与阀口的开度 大小成线性关系，因此通过安装在开关阀左端上的 非接触式电浴流位移传感器, 采集开关阀的阀芯位 移信号, 来测试开关阀的动态启、闭特性。

\section{2 静态流量特性测试}

采用 PWM 驱动方式, 依次测试不同占空比下, 压电开关阀的平均流量, 得到其流量-占空比曲线; 改变 PWM 载波频率, 可得到不同载波频率 $(10 \mathrm{~Hz}$ 、 $100 \mathrm{~Hz} 、 500 \mathrm{~Hz})$ 下, 高速开关阀的流量-占空比曲 线如图 14 所示。

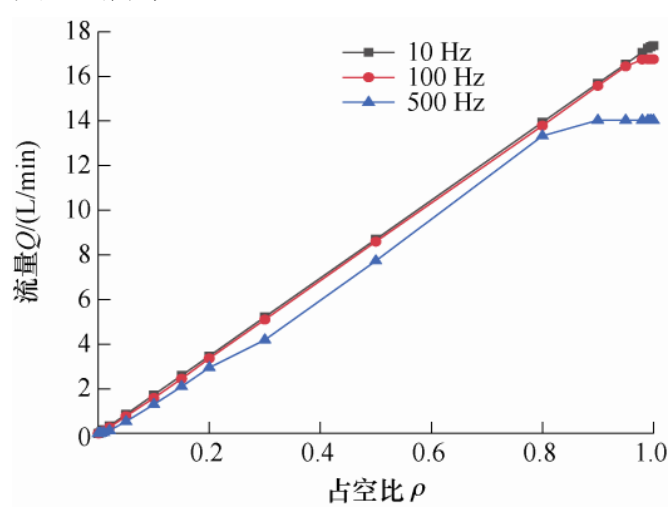

图 14 不同 PWM 载波频率下流量-占空比曲线

由图 14 所示的流量-占空比曲线可以看出: 高 速开关阀稳态最大流量 $16.9 \mathrm{~L} / \mathrm{min}$ ，和仿真结果基 本一致; 采用 PWM 控制方式, 在一定载波频率下, 压电式高速开关阀流量与占空比基本呈线性关系, 但仍然存在较小的死区、非线性区和饱和区; 随着 PWM 载波频率的增加, 流量曲线的死区, 非线性 区和饱和区宽度增大, 而线性区将减小 $(f=10 \mathrm{~Hz}$, $\rho$ 在 $0.7 \% \sim 99 \% ; f=100 \mathrm{~Hz}, \rho$ 在 $5 \% \sim 96 \%$; $f=500 \mathrm{~Hz}, \rho$ 在 $25 \% \sim 76 \%$ )。

\section{3 动态开关特性测试}

取 PWM 信号载波频率 $f=100 \mathrm{~Hz}$, 占空比 $\rho=0.5$, 分别通过压电叠堆自带的应变式传感器、 电浴流传感器测试压电执行器和开关阀的阀芯位移 曲线如图 15 所示。

图 15 中, 纵轴代表位移(流量), 横轴代表时间。 由位移(流量)曲线可以看出: 压电执行器输出最大 位移 $26 \mu \mathrm{m}$, 经位移放大机构后, 阀芯位移 $110 \mu \mathrm{m}$, 位移放大倍数 4.23 ; 压电执行器位移小于空载最大 位移 $(30 \mu \mathrm{m})$, 主要是由零位装配时, 位移放大结构 即复位弹簧的预压力和阀芯运动时的液压力决定; 阀芯完全开启时间为 $1.15 \mathrm{~ms}$; 阀门基本完全关闭 所需时间 $0.85 \mathrm{~ms}$, 与仿真结果基本一致。 


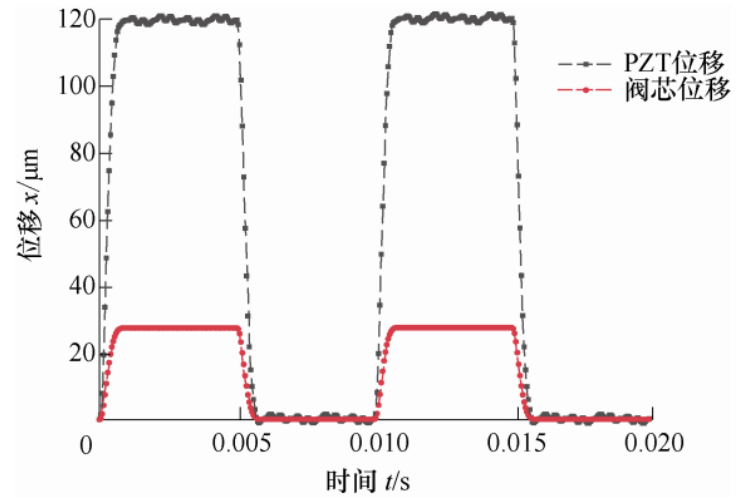

图 15 开关阀启、闭试验曲线

仿真测试 PWM 载波频率 $f=100 \mathrm{~Hz}$ 时, 不同 占空比下阀芯位移(瞬态流量)曲线如图 16 所示。
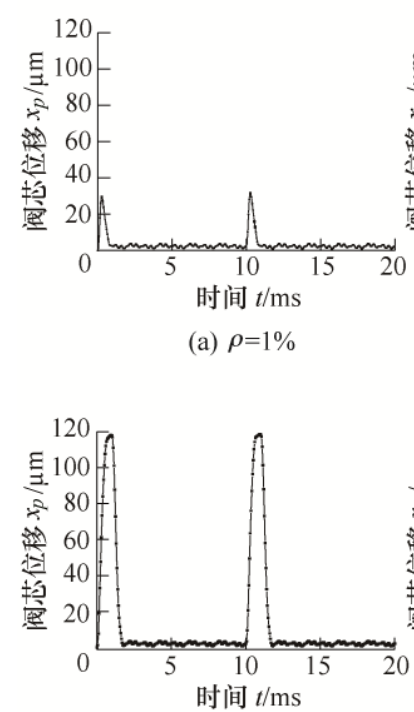

(c) $\rho=10 \%$

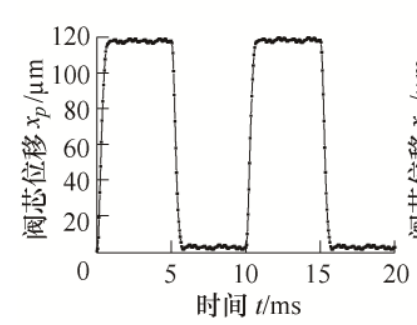

(e) $\rho=50 \%$

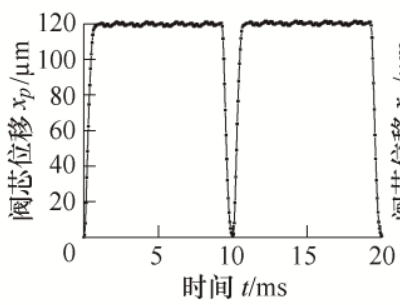

(g) $\rho=94 \%$ (a) $\rho=1 \%$

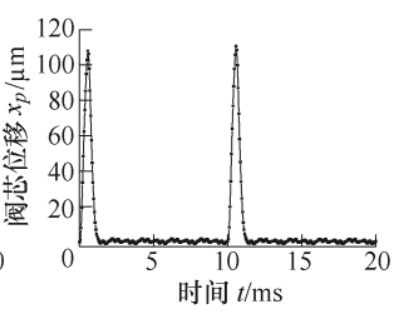

(b) $\rho=5 \%$

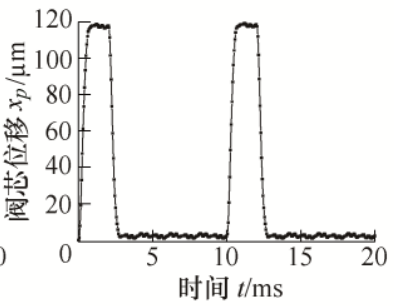

(d) $\rho=20 \%$

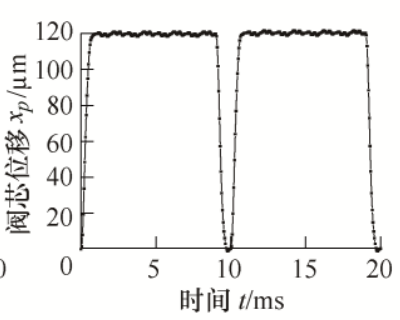

(f ) $\rho=90 \%$
图 16 不同占空比下阀芯位移(瞬态流量)测试曲线

通过图 16 所示测试结果可以看出, 当占空比非
常小, 开启脉冲时间小于死区时间时, 阀口来不及 打开，控制流量基本为零(死区段); 随着占空比的 逐渐增加, 阀口打开程度逐渐增大, 阀口可控流量 非线性增加(非线性段), 当 $\rho \geqslant 0.1$ 时即可保证阀口 完全打开，此后阀口控制流量与占空比基本呈线性 (线性段), 直到 $\rho=0.94$; 当 $\rho>0.94$ 时, 无法保证 关闭过程中阀口的完全关闭(饱和区)。

\section{4 试验结果分析}

结合图 14 16 所示开关阀静态及动态特性测 试曲线可以看出以下几点。

（1）在线性段，PWM 波频率一定条件下，阀口 平均流量 $\bar{q}$ 与 $\mathrm{PWM}$ 波占空比 $\rho$ 之间的关系(假设 $q_{\text {max }}$ 为阀口全开时的流量, 用平均流量 $\bar{q}$ 近似表示 其一个周期内的平均流量)

$$
\bar{q}=\frac{t_{\text {on }}}{t_{\text {on }}+t_{\text {off }}} q_{\text {max }}
$$

式中, $t_{\mathrm{on}}$ 表示高速开关阀在一个周期内的开启时间, $t_{\text {off }}$ 表示其一个周期内的关闭时间, 式 $t_{\text {on }} /\left(t_{\text {on }}+t_{\text {off }}\right)$ 即为占空比 $\rho$ 。

(2) 随着 PWM 载波频率的增加, 流量曲线的死 区，非线性区和饱和区宽度增大，而线性区减小。 主要原因是压电执行器驱动的阀芯运动系统, 其机 电响应时间是固定的，因此开关阀的流量响应时间 是确定的; 当载波频率较小时, 载波周期大, 机电 响应时间相比载波周期时间, 所占的比值较小，所 以占空比小, 系统的死区、非线性区和饱和区较小; 随着载波频率的增加, 载波周期减小, 机电响应时 间相比载波周期所占的比值增加, 所以相应占空比 增大，系统的死区、非线性区和饱和区均增大。

(3) 非线性区内阀口未完全开启, 理论上对应 开关阀的流量分辨率增加, 但流量控制精度的重复 性及可靠性较差。

\section{5 结论}

设计基于桥式位移放大结构的压电直动式高速 开关阀, 实现快速性与大流量的需求, 对优化设计 的压电式开关阀静、动态流量及特性进行仿真及分 析、测试, 结论如下。

（1）压电式开关阀响应迅速，其开启时间 $1.15 \mathrm{~ms}$, 关闭时间 $0.85 \mathrm{~ms}$, 启闭性能优于常见的 电磁式开关阀。

（2）设计柔性铰链桥式位移放大结构增加了开 关阀的流量，最大流量可达 $17.4 \mathrm{~L} / \mathrm{min}$ 。

(3) 在一定范围内, 开关阀的流量与 PWM 波的 
占空比基本成线性关系(线性区范围由载波频率大 小决定), 通过调节占空比的大小可以有效调节开关 阀的平均流量, 其分辨率可达 $0.75 \%$ 。

(4) 由于压电执行器具有迟滞特性, 开关阀具 有 $0.2 \mathrm{~ms}$ 的死区, $0.8 \mathrm{~ms}$ 的非线性区以及 $0.85 \mathrm{~ms}$ 的饱和区; 下一步工作将研究相对应的智能控制和 驱动方法, 补偿其死区和饱和区特性, 合理利用非 线性区特性, 进一步提升开关阀的响应速度和流量 分辨率。

\section{参 考 文 献}

[1] 路角祥. 流体传动与控制技术的历史进展与展望 $[\mathrm{J}]$. 机械工程学报, 2001, 37(10): 1-9.

LU Yongxiang. Historical progress and prospects of fluid power transmission and control[J]. Journal of Mechanical Engineering, 2001, 37(10): 1-9.

[2] 杨华勇, 王双, 张斌, 等. 数字液压阀及其阀控系统发 展和展望[J]. 吉林大学学报, 2016, 46(5): 1494-1505. YANG Huayong, WANG Shuang, ZHANG Bin, et al. Development and prospect of digital hydraulic valve and valve control system[J]. Journal of Jilin University, 2016, 46(5): 1494-1505.

[3] 丁凡, 姚健娣, 笪靖, 等. 高速开关阀的研究现状[J]. 中 国工程机械学报, 2011，9(3): 351-358.

DIN G Fan, YAO Jiandi, DA Jing, et al. Advances on high- speed on-off valves[J]. Chinese Journal of Construction Machineny, 2011, 9(3): 351-358.

[4] NIE Songlin, LIU Xiangyang, YIN Fanglong, et al. Development of a high-pressure pneumatic on/off valve with high transient performances direct-driven by voice coil motor[J]. Applied Sciences-Basel, 2018, 8(4): 611.

[5] YANG Zhaoshu, HE Zhongbo, LI Dongwei, et al. Direct drive servo valve based on magnetostrictive actuator: Multi-coupled modeling and its compound control strategy[J]. Sensors and Actuators A-Physical, 2015, 235: 119-130.

[6] FANG Jingen, WANG Xifeng, WU Jinjun, et al. Modeling and control of a high speed on/off valve actuator[J]. International Journal of Automotive Technology, 2019, 20(6): 1221-1236.

[7] WU Shuai, ZHAO Xiangyu, LI Chunfang, et al. Multiobjective optimization of a hollow plunger type solenoid for high speed on/off valve[J]. IEEE Transactions on Industrial Electronics, 2018, 65(4): 3115-3124.

[8] ZHANG Bin, ZHONG Qi, MA J E, et al. Self-correcting
PWM control for dynamic performance preservation in high speed on/off valve[J]. Mechatronics, 2018, 55: 141-150.

[9] JEON J, NGUYEN Q H, HAN Y M, et al. Design and evaluation of a direct drive valve actuated by piezostack actuator[J]. Advances in Mechanical Engineering, 2013: 986812.

[10] 许有熊, 朱青松. 压电数字阀电-机械转换器设计与分 析[J]. 机械设计，2013，30(11): 77-82.

XU Youxiong, ZHU Qingsong. Design and analyze of piezoelecreic digital valve mechanical converter[J]. Journal of Machine Design, 2013， 30(11): 77-82.

[11] 阮健, 裴翔, 李胜. $2 \mathrm{D}$ 电液数字换向阀 [J]. 机械工程学 报，2000，36(3): 86-89.

RUAN Jian, PEI Xiang, LI Sheng. 2D digital directional control valve[J]. Journal of Mechanical Engin- eering, 2000, 36(3): 86-89.

[12] ZHU Muzhi, ZHAO Shengdun, DONG Peng, et al. Design and analysis of a novel double-servo direct drive rotary valve with high frequency[J]. Journal of Mechanical Science and Technology, 2018，32(9): 4313-4323.

[13] 江海兵, 阮健, 李胜, 等. $2 \mathrm{D}$ 电液高速开关阀设计与 实验 $[\mathrm{J}]$. 农业机械学报，2015，46(2)：328-334.

JIANG Haibing, RUAN Jian, LI Sheng, et al. Design and experiment of 2D electrohydraulic high-speed on-off valve[J]. Transactions of the Chinese Society for Agricultural Machinery, 2015, 46(2): 328-334.

[14] 陆豪, 朱成林, 曾思, 等. 新型 PZT 元件驱动的电液 高速开关阀及其大功率快速驱动技术的研究 $[\mathrm{J}]$. 机械 工程学报，2002，38(8): 118-121.

LU Hao, ZHU Chenglin, ZENG Si, et al. Study on new kind of electro-hydraulic high-speed on-off valve driven by PZT components and its high-powerful and speedy technique[J]. Journal of Mechanical Engineering, 2002, 38(8): 118-121.

[15] 石延平, 刘成文, 张永忠. 一种大流量高速开关阀的研 究与设计[J]. 机械工程学报, 2004, 40(4): 195-198.

SHI Yanping, LIU Chengwen, ZHANG Yongzhong. Design and study of a new kind of large flow rate highspeed on-off valve[J]. Journal of Mechanical Engineering, 2004, 40(4): 195-198.

[16] 沈传亮, 程光明, 杨志刚, 等. 新型直动式压电伺服阀 [J]. 机械工程学报, 2004, 40(9): 125-128.

SHEN Chuanliang, CHENG Guangming, YANG Zhigang, et al. New type piezoelectric direct drive servo 
valve[J]. Journal of Mechanical Engineering, 2004, 40(9): 125-128.

[17] 俞军涛, 焦宗夏, 吴帅. 基于液压微位移放大结构的新 型压电陶瓷直接驱动阀设计及仿真 $[\mathrm{J}]$. 机械工程学报, 2013, 49(2): 151-158.

YU Juntao, JIAO Zongxia, WU Shuai. Design and simulation study on new servo valve direct driven by piezoelectric actuator using hydraulic amplification[J]. Journal of Mechanical Engineering, 2013, 49(2): 151-158.
[18] 欧阳小平, 杨华勇, 蒋吴宜, 等. 新型压电高速开关阀 仿真研究[J]. 科学通报, 2008, 53(14): 1737-1741. OUYANG Xiaoping, YANG Huayong, JIANG Haoyi, et al. Simulation of high-speed switching valve with new typed piezoelectric[J]. Chinese Science Bulletin, 2008, 53(14): 1737-1741.

作者简介: 俞军涛, 男, 1982 年出生, 博士, 讲师。主要研究方向为机 电液伺服元件及系统设计、仿真、控制。

E-mail: Juntaoyu_vip@163.com 